\title{
Estudo de casos múltiplos das parcerias para o desenvolvimentoprodutivo: doenças negligenciadas versus doenças crônicas não transmissíveis.
}

\author{
Productive development partnerships multiple \\ cases study: neglected diseases versus non- \\ communicable chronic diseases.
}

\section{Estudio de los múltiples casos de las asociaciones para el desarrollo productivo: enfermidades desatendidas frente a las enfermidades crónicas no transmisibles.}

Gabriela de Oliveira Silva ${ }^{1}$

Flávia Tavares Silva Elias ${ }^{1}$

\begin{abstract}
RESUMO:As Parcerias para o Desenvolvimento Produtivo (PDP) são uma das iniciativas brasileiras para enfrentamento das dificuldades de acesso a medicamentos considerando a concomitância de doenças negligenciadas e doenças crônicas não transmissíveis no Brasil. Este estudo teve como objetivo compreender se essa iniciativa, no formato atual, está adequada para a garantia do abastecimento de medicamentos para doenças negligenciadas. Buscou-se identificar os elementos que influenciam a execução das parcerias e a apresentação de novas propostas, comparando-se PDP de medicamentos de doença infecciosa negligenciada, tuberculose, e crônica não transmissível, esclerose múltipla. Foi realizado um estudo de casos múltiplos, utilizando pesquisas documental e da literatura, e inquérito com aplicação de questionários eletrônicos a atores envolvidos com as PDP firmadas desde 2009 e vigentes em 2016, seguido de análise de conteúdo. Os resultados demonstraram que os elementos que influenciam a execução das PDP e a apresentação de novas propostas estão associados a aspectos econômicos e sociais das tecnologias e das patologias que
\end{abstract}

1 Fundação Oswaldo Cruz

ISSN 1982-8829 Tempus, actas de saúde colet, Brasília, 11(4), 147-169, dez, 2017. Epub Ago/2018 
visam tratar. $\mathrm{O}$ modelo de PDP atual pode não ser a estratégia mais adequada para a garantia do abastecimento de medicamentos para doenças negligenciadas. Assim, podem ser necessárias adequações no modelo atual, como as identificadas pelos atores envolvidos com a iniciativa especialização de laboratórios públicos em plataformas produtivas; conjugação de produtos de alto e baixo valor agregado e articulação a outros incentivos financeiros - ou a implantação de novas estratégias, como o desenvolvimento de PDP de pesquisa, desenvolvimento e inovação.

Palavras-chave: Parcerias público-privadas, Doença negligenciada, Doença crônica.

ABSTRACT: Productive development partnerships (PDP) are one of the Brazilian initiatives for the combat against the difficulties in access to medicines, considering the concomitance of neglected diseases and non-communicable chronic diseases in Brazil. The objective in this study was to comprehend if this initiative, in the current format, is suitable to guarantee the supply of medicines for neglected diseases. There was an effort to identify the elements that influence the execution of the partnerships and the presentation of new proposals, comparing PDP associated with medicines for a neglected infectious disease, tuberculosis, and for a non-communicable chronic disease, multiple sclerosis. A multiple case study was carried out, using documentary and literature research, and a inquiry with electronic surveys applied to actors involved in PDP consolidated in 2009 and active in 2016, followed by content analysis. The results showed that the elements that influence PDP execution and the presentation of new proposals are associated with economic and social aspects regarding the technologies and diseases they aim to treat. The current PDP model might not be the most adequate strategy to ensure the supply of medicines for neglected diseases. Thus, adaptations in the current model may be need, such as the ones identified by the actors involved with the initiative - the specialization of public laboratories in productive platforms; the combination of products with high and low added value and the articulation with other financial incentives - or the implementation of new strategies, such as the development of research, development and innovation PDP.

Keywords: Public-Private Sector Partnerships, Neglected disease, Chronic disease.

RESUMEN: Las Asociaciones para el Desarrollo Productivo (PDP) es una de las iniciativas brasileñas para hacer frente a las dificultades de acceso a los medicamentos teniendo encuenta las enfermedades desatendidas y las enfermedades crónicas no transmisibles en Brasil. Este estudio tuvo como objetivo comprender si esta iniciativa, ensu forma actual, es suficiente para garantizar el suministro de medicamentos para enfermedades olvidadas. Hemos tratado de identificar los elementos que influyenen la aplicación de las asociaciones y la presentación de nuevas propuestas, comparando uma PDP de medicamento para tuberculosis, una enfermedad infecciosa, y una PDP de medicamento para esclerosis múltiple, una enfermedad crónica no transmisible. Se realizó un estudio de casos múltiples utilizando análisis de documentos, indagación de los actores involucrados en el PDP firmadas desde el año 2009 y en vigor en 2016, yanálisis de contenido. Los resultados mostraron que los factores que influyen en el rendimiento del PDP y la presentación de nuevas propuestas están asociados con los aspectos económicos y sociales de las tecnologías y las

Tempus, actas de saúde colet, Brasília, 11(4), 147-169, dez, 2017. Epub Ago/2018 ISSN 1982-8829 
enfermidades. El modelo PDP actual puede no ser la estrategia más adecuada para la seguridad del suministro de medicamentos para enfermedades desatendidas. Por lo tanto, pueden ser necesarios ajustes en el modelo actual, identificados por los actores que participan en la iniciativa - la especialización de laboratorios públicos en plataformas productivas; la combinación de productos de alto y bajo valor agregado y la articulación a otros incentivos financieros -, o la implementación de nuevas estrategias como el desarrollo de PDP de investigación, desarrollo e innovación.

Palabras clave: Asociaciones entre el Sector Público y el Privado, Enfermedadesdesatendidas, Enfermedad crónica.

\section{INTRODUÇÃO}

As transições demográficas, epidemiológicas e nutricionais no Brasil impactaram diretamente nas alterações das taxas de morbidade e mortalidade ${ }^{1}$, conduzindo a uma situação de saúde no país de tripla carga de doenças: problemas de saúde reprodutiva; crescimento das causas externas e predomínio das doenças crônicas não transmissíveis (DCNT); e manutenção das doenças infecciosas e parasitárias, consideradas negligenciadas por não serem prioridade para produção de medicamentos e insumos ${ }^{2,3}$.

Doenças negligenciadas (DN) correspondem a um grupo diversificado de infecções causadas por agentes patogênicos, tais como vírus, bactérias, protozoários e helmintos ${ }^{4}$. São doenças que prevalecem em condições de pobreza e contribuem para a manutenção do quadro de desigualdade ${ }^{5}$. Podem influenciar desfavoravelmente no crescimento infantil, no desenvolvimento intelectual e na produtividade do trabalho ${ }^{6}$. Dengue, doença de Chagas, leishmaniose, malária, esquistossomose, hanseníase e tuberculose são DN para o Brasil ${ }^{7}$.

Segundo a Organização Mundial da Saúde(OMS), pelo menos 1,7 bilhão de pessoas necessitavam de tratamento para DN em 185 países em $2014^{8}$. Desse número, 1,1 bilhão de pessoas estavam em países de renda média-baixa e 520 milhões em países de baixa renda, representando este último $60 \%$ de suas populações ${ }^{8}$.

Entretanto, os investimentos em pesquisa e desenvolvimento de novos fármacos para essas enfermidades ainda são reduzidos. Das 1.556 novas entidades químicas comercializadas mundialmente de 1975 a 2004, apenas 10 correspondem a medicamentos para o tratamento de DN, considerando-se as doenças mais negligenciadas; 18, adicionando-se a malária; e 21, considerandose também a tuberculose ${ }^{6}$. Doenças mais negligenciadas incidem, principalmente, nos países em desenvolvimento, sendo os incentivos para pesquisa e desenvolvimento praticamente inexistentes nestes países, bem como praticamente não são objeto de pesquisa pelos países desenvolvidos ${ }^{7}$.

Quanto às DCNT, estas estão relacionadas a causas múltiplas, são caracterizadas por início 
gradual, de prognóstico usualmente incerto, com longa ou indefinida duração, podendo gerar incapacidades e exigindo cuidado contínuo. Acidente vascular cerebral, hipertensão arterial, câncer, diabetes e doenças respiratórias crônicas são exemplos de $\mathrm{DCNT}^{9}$. Tais doenças, em sua totalidade, foram a causa de cerca de 38 milhões de mortes no ano de 2012, o que representou $68 \%$ das mortes em todo o mundo ${ }^{8}$. Das mortes prematuras, ou seja, antes dos 70 anos, aproximadamente $52 \%$ foram decorrentes de DCNT, sendo três quartos devido a doenças cardiovasculares, câncer, diabetes e doenças respiratórias crônicas ${ }^{8}$. As duas primeiras apresentam maior proporção em termos de mortalidade prematura, que atingiu 10 milhões de pessoas 8 .

Diante desse contexto, medidas relacionadas às DN e DCNT foram incluídas entre as metas do Objetivo 3 do Desenvolvimento Sustentável, estabelecido em 2015 no âmbito das Organizações Unidas baseado nos Objetivos do Desenvolvimento do Milênio. Tal objetivo trata de "Assegurar uma vida saudável e promover o bem-estar para todos, em todas as idades" ${ }^{10}$. As metas são de, até 2030, acabar com as epidemias de DN e reduzir em um terço a mortalidade prematura por DCNT via prevenção e tratamento.

No âmbito do Sistema Único de Saúde (SUS), baseando-se nos princípios de universalidade, integralidade e equidade, diversas estratégias de saúde pública são adotadas para a prevenção e o tratamento das DN e DCNT. Entre estas, destacam-se a Política Nacional de Medicamentos (PNM), criada em 1998, e a Política Nacional de Assistência Farmacêutica (PNAF) que a substituiu em 2004. Tais Políticas têm o propósito de garantir à população medicamentos essenciais, envolvendo as três esferas de governo.

Por meio do Bloco de Financiamento da Assistência Farmacêutica são fornecidos à população medicamentos e insumos, conforme protocolos clínicos e diretrizes terapêuticas. Esse Bloco é constituído por três componentes: i) componente básico da assistência farmacêutica (CBAF); ii) componente estratégico da assistência farmacêutica (CESAF); e iii) componente especializado da assistência farmacêutica (CEAF) ${ }^{6}$.

Os medicamentos para DN estão incluídos no $\mathrm{CESAF}^{6}$ e, assim como os medicamentos para DCNT do Grupo 1A do CEAF, têm sua aquisição centralizada pelo Ministério da Saúde (MS) e são repassados para os Estados para armazenamento e distribuição aos Municípios.

Entretanto, são grandes os desafios impostos ao Estado brasileiro para garantia do acesso universal desses medicamentos à população. Entres eles, estão a defasagem tecnológica associada à dependência produtiva nacional; demanda crescente da população; predomínio de empresas multinacionais em ambiente de oligopólio ${ }^{11,12}$; recursos orçamentários reduzidos frente aos preços dos produtos e ao montante necessário; vulnerabilidade do SUS frente ao mercado internacional ${ }^{13}$; e o baixo interesse da indústria farmacêutica em produtos para o tratamento de $\mathrm{DN}^{14}$. 
Como apontado por Garcia et al. ${ }^{6}$, na era da ciência e da tecnologia, enquanto direitos humanos, causas e consequências das iniquidades em saúde são pontos fortes de discussão, populações pobres não têm acesso ao tratamento de $\mathrm{DN}$, sendo necessárias ações para o desenvolvimento de medicamentos e vacinas eficientes e acessíveis.

Para enfrentar esses desafios, estratégias vêm sendo adotadas pelo Estado brasileiro por meio da articulação de políticas de saúde, industrial e de ciência, tecnologia e inovação para o desenvolvimento da base produtiva nacional, inserida no Complexo Econômico Industrial da Saúde (CEIS). Entre estas, destacam-se as Parcerias para o Desenvolvimento Produtivo (PDP) coordenadas pelo MS para a transferência de tecnologia de empresas privadas e capacitação de produtores públicos para o atendimento das demandas do SUS.

De 2009 a 2016, 104 PDP foram estabelecidas, as quais abarcaram diferentes tipos de produtos - vacinas (29\%), medicamentos $(65 \%)$ e produtos para saúde $(6 \%)$ e, portanto, envolvem níveis de complexidade distintos de transferência e absorção de tecnologia ${ }^{15}$. As indicações terapêuticas dos produtos também são variadas, incluindo produtos para doenças raras, DN e DCNT.

O processo de estabelecimento das PDP inclui quatro fases: I - submissão e análise da proposta e, em caso de aprovação, celebração do termo de compromisso (TC) entre o MS e o produtor público; II - execução do projeto com início da implementação do TC; III - desenvolvimento do produto, transferência e absorção de tecnologia e aquisição do produto pelo MS; e IV - internalização de tecnologia pelo produtor público ${ }^{16}$.

Esse processo é permeado por diversos elementos intrínsecos ou externos que dificultam a execução e a conclusão dos projetos. Tanto é que 23 parcerias foram extintas de 2014 a 2016 : 13 de medicamentos de síntese química, sendo três para DN; 8 biológicos para DCNT, sendo um referente a produto órfão; e 2 de produtos para saúde. Acredita-se que tais elementos são variáveis de acordo com o tipo de produto, da rota de síntese do fármaco, da indicação terapêutica e dos aspectos econômicos e sociais envolvidos.

Verifica-se também um cenário diferenciado no âmbito das PDP de medicamentos para DN. Desde o lançamento da iniciativa, foram estabelecidas quatro parcerias de produtos para DN, o que representa aproximadamente $4 \%$ do total de PDP firmadas no período. Destas, apenas a PDP de Rifampicina, Isoniazida, Etambutol e Pirazinamida (4 em 1) firmada em 2009 pelo Instituto de Tecnologia em Fármacos (Farmanguinhos) da Fundação Oswaldo Cruz (Fiocruz) encontra-se vigente $^{15}$. Os outros três projetos foram extintos em 2015 e 2016: Anfotericina B Lipossomal para leishmaniose visceral, Desoxicolato de Anfotericina B para o tratamento de pacientes coinfectados leishmania/HIV e Difosfato de Cloroquina para malária, estabelecidos em 2013 pelo Laboratório Farmacêutico do Estado do Rio Grande do Sul (LAFERGS) em parceria com o Cristália Produtos 
Químicos Farmacêuticos ${ }^{15}$.

Em contrapartida, PDP de medicamentos para DCNT preponderam no total de parcerias. A exemplo, para os produtos biológicos, verificou-se grande competitividade entre as instituições proponentes em 2015: sete propostas de PDP apresentadas para Adalimumabe, quatro para Filgrastim, seis para Infliximabe, quatro para Rituximabe e três para Somatropina ${ }^{17}$.

Diante desse contexto, esta pesquisa teve como objetivo compreender se a iniciativa das PDP, no formato atual, está adequada para a garantia do abastecimento de medicamentos para DN. Assim, buscou-se identificar os elementos que influenciam a execução das parcerias e a apresentação de novas propostas, comparando-se PDP de medicamentos para DN e DCNT.

\section{MÉTODO}

Esta pesquisa traz uma abordagem qualitativa e envolve o estudo de casos múltiplos, o qual se caracteriza por estudar simultaneamente mais de um caso particular representativo de um conjunto de casos análogos por eles significativamente representativos $(18,19)$. Trata-se de "uma investigação empírica que investiga um fenômeno contemporâneo dentro de seu contexto da vida real, especialmente quanto aos limites entre o fenômeno e o contexto que não estão claramente definidos", buscando conhecer o como e o porquê de uma situação específica ${ }^{19}$.

A Figura 1 representa graficamente o método de desenvolvimento do estudo de casos múltiplos das PDP, realizado com base no modelo proposto por Yin ${ }^{19}$.

Figura 1 - Método de desenvolvimento do estudo de casos múltiplos das PDP

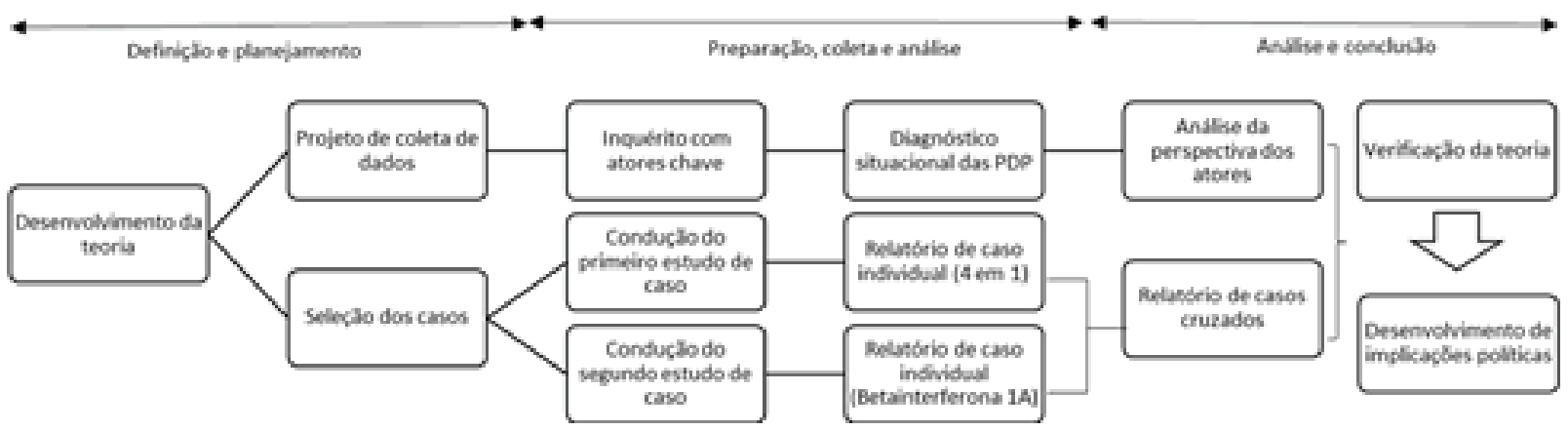

Fonte: adaptado de Cosmos Corporation apud Yin ${ }^{19}$. 
Uma vez que o estudo de casos múltiplos é baseado em várias fontes de evidências ${ }^{19}$, a pesquisa de campo, por meio de inquérito, foi articulada às pesquisas documental e bibliográfica para coleta de evidências que buscassem verificar a seguinte teoria: a iniciativa das PDP, no formato atual, não está adequada para a garantia do abastecimento de medicamentos para DN, tendo em vista as diferenças que ocorreram com as parcerias durante 2009 a 2016.

O inquérito foi realizado de setembro a novembro de 2016 junto aos atores-chave envolvidos com a gestão das PDP de medicamentos implementadas de 2009 a 2016 e envolveu um escopo maior de pesquisa. Para este estudo, foram utilizadas as respostas a três perguntas do questionário, as quais buscaram identificar, na perspectiva dos entrevistados, os elementos dificultadores e facilitadores para execução de projetos de medicamentos para DN e para DCNT, bem como as medidas que poderiam auxiliar no processo de estabelecimento das parcerias para cada grupo desses medicamentos.

Os questionários eletrônicos foram elaborados na plataforma FormSUS e aplicados a 81 atores: 18 representantes de produtores públicos e 34 de entidades privadas; e 29 membros da Comissão Técnica de Avaliação (CTA), do Comitê Deliberativo (CD), e da coordenação dos Comitês Técnico Regulatórios (CTR). O pré-teste desse instrumento de pesquisa foi realizado por um gestor público e um pesquisador quanto à objetividade e clareza das questões, à coerência e ao tempo de preenchimento. A análise dos dados obtidos foi feita de forma narrativa e sistematizada em quadro comparativo.

De forma a identificar mais evidências de como e por que os elementos e medidas apresentados influenciam o processo de estabelecimento das parcerias na realidade, foram selecionadas duas PDP para um estudo de comparabilidade. Os critérios de seleção dos dois casos foram: a) uma PDP de cada grupo de medicamentos, quais sejam DN e DCNT; b) aprovação das propostas antes do primeiro marco normativo específico das parcerias, ou seja, de 2009 a 2012; c) duração da fase II entre cinco e seis anos; d) mesma data de início da fase III e, portanto, que se encontravam em 2016 no mesmo ano desta fase; e) rotas produtivas dos insumos farmacêuticos ativos (IFA) e dos medicamentos distintas e associadas a diferentes tecnologias e custos - aspectos econômicos e mercadológicos; f) formas farmacêuticas diferentes; e g) classe terapêutica e aspectos sociais envolvidos diversos.

Por esses critérios, foram selecionadas para o estudo as parcerias dos seguintes medicamentos: Rifampicina, Isoniazida, Etambutol e Pirazinamida (4 em 1) para tuberculose; e Betainterferona 1A para esclerose múltipla. As semelhanças entre as parcerias selecionadas possibilitaram condições de comparabilidade do processo de estabelecimento das mesmas de forma a permitir a discussão da influência dos aspectos divergentes no monitoramento desse processo. 
A coleta de dados específicos sobre essas PDP foi realizada utilizando as variáveis para a pesquisa, os documentos e bases de dados indicados no Quadro 1.

Quadro 1- Variáveis, documentos e bases de dados consultados para elaboração do estudo de casos múltiplos das Parcerias para o Desenvolvimento Produtivo (PDP)

\begin{tabular}{|c|c|}
\hline Variáveis & Documentos e bases de dados consultados \\
\hline $\begin{array}{l}\text { Nome, grupo, indicaçãoterapêutica, forma farmacêutica } \\
\text { e apresentação dos medicamentos; produtor público e } \\
\text { parceiros desenvolvedores dos insumos farmacêuticos } \\
\text { ativos e dos medicamentos nas PDP; ano de aprovação } \\
\text { das propostas; fase do processo em } 2016 \text {. }\end{array}$ & $\begin{array}{l}\text { Propostas de Projetos de PDP aprovadas de } 2009 \text { a } \\
2014 \text { (planilha). Portal eletrônico do MS: www.saude. } \\
\text { gov.br/deciis }\end{array}$ \\
\hline Rota de síntese dos insumos farmacêuticos ativos. & $\begin{array}{l}\text { Listas de produtos estratégicos para o Sistema Único } \\
\text { de Saúde (SUS): Portarias GM/MS no } 978 \text {, de } 16 \text { de } \\
\text { maio de } 2008, \text { e } n^{\circ} 1.284 \text {, de } 26 \text { de maio de } 2010 \text {. Portal } \\
\text { eletrônico do MS: www.saude.gov.br/deciis }\end{array}$ \\
\hline $\begin{array}{l}\text { Data de início da fase III que corresponde à publicação } \\
\text { do primeiro instrumento específico de compra do } \\
\text { produto pelo MS no âmbito da PDP. } \\
\text { Valores da primeira e segunda aquisições dos } \\
\text { medicamentos pelo MS. }\end{array}$ & $\begin{array}{l}\text { Aquisições de produtos de Parcerias para o } \\
\text { Desenvolvimento Produtivo (PDP) (planilha). Portal } \\
\text { eletrônico do MS: www.saude.gov.br/deciis. } \\
\text { Extratos dos Termos de Execução Descentralizada. } \\
\text { Diário Oficial da União (DOU). }\end{array}$ \\
\hline $\begin{array}{l}\text { Datas de obtenção dos registros sanitários dos } \\
\text { medicamentos na Agência Nacional de Vigilância } \\
\text { Sanitária (ANVISA) pelos parceiros privado e público }\end{array}$ & $\begin{array}{l}\text { Consulta de medicamentos. Portal eletrônico da } \\
\text { ANVISA. }\end{array}$ \\
\hline $\begin{array}{l}\text { Linhas produtivas e validade dos Certificados de Boas } \\
\text { Práticas de Fabricação (CBPF) }\end{array}$ & Consulta CBPF. Portal eletrônico da ANVISA. \\
\hline Situação de entrega do produto da PDP & $\begin{array}{l}\text { Questionamento enviado pelo Sistema eletrônico de } \\
\text { Informação ao Cidadão (e-SIC). }\end{array}$ \\
\hline
\end{tabular}

Fonte: elaboração própria (2016).

O protocolo desta pesquisa foi aprovado pelo Comitê de Ética em Pesquisa da Fiocruz Brasília no sistema Plataforma Brasil conforme Parecer n ${ }^{\circ}$ 1.549.078, de 17 de maio de 2016. Este estudo faz parte da dissertação de mestrado da primeira autora, intitulada "Parcerias para o Desenvolvimento Produtivo e a produção pública de medicamentos: uma proposta de monitoramento estratégico".

\section{RESULTADOS}

Dos entrevistados, 14, 33 e 17 responderam as questões sobre elementos facilitadores, dificultadores e medidas auxiliares ao processo de estabelecimento das PDP, respectivamente. Isto representa uma taxa média de resposta de $26 \%$. O Quadro 2 demonstra de forma sistematizada tais elementos e medidas de acordo com a perspectiva dos atores envolvidos com as PDP. 
Quadro 2 - Elementos facilitadores e dificultadores e medidas auxiliares ao processo de estabelecimento das Parcerias para o Desenvolvimento Produtivo (PDP) na perspectiva dos atores evolvidos com as parcerias

\begin{tabular}{|c|c|c|}
\hline $\begin{array}{l}\text { Elementos facilitadores } \\
\qquad(n=14)\end{array}$ & $\begin{array}{l}\text { Elementos dificultadores } \\
\qquad(\mathrm{n}=33)\end{array}$ & $\begin{array}{l}\text { Medidas auxiliares } \\
(\mathrm{n}=17)\end{array}$ \\
\hline 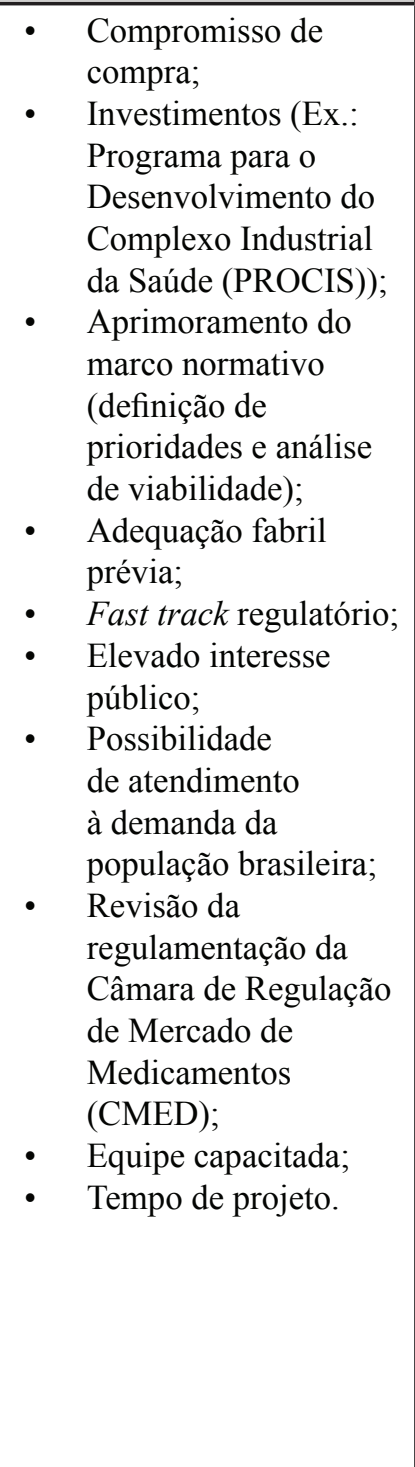 & 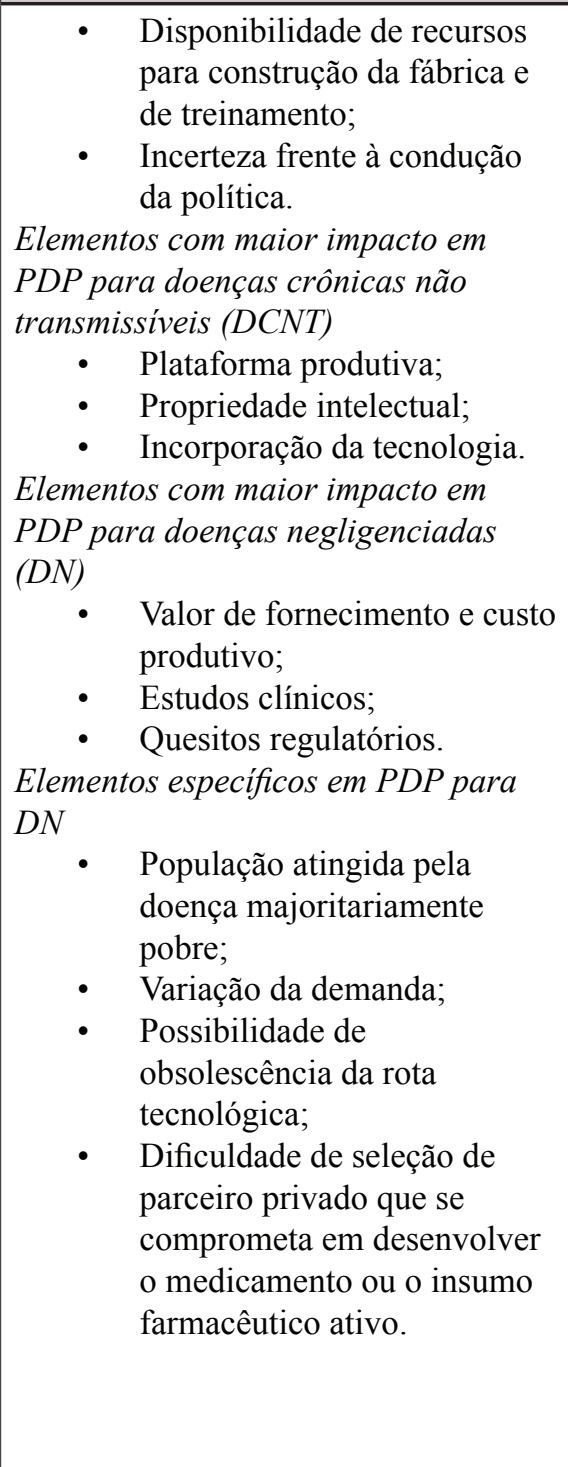 & $\begin{array}{l}\text { - Vinculação de produtos de baixo e } \\
\text { de alto valor agregado; } \\
\text { - } \quad \text { Definição de listas mais amplas; } \\
\text { Especialização dos produtores } \\
\text { públicos por plataforma } \\
\text { tecnológica; } \\
\text { - Segurança para as entidades } \\
\text { visto o alto nível de investimento } \\
\text { requerido (tempo e recursos); } \\
\text { Garantia do cumprimento das } \\
\text { responsabilidades do gestor } \\
\text { federal (ex.: definição do início de } \\
\text { aquisição); } \\
\text { Avaliação do horizonte } \\
\text { temporal de substituição da rota } \\
\text { tecnológica; } \\
\text { Precificação diferenciada e } \\
\text { investimentos do Ministério da } \\
\text { Saúde para medicamentos para } \\
\text { DN; } \\
\text { Auxílio financeiro para os } \\
\text { produtores públicos para } \\
\text { investimentos em tecnologia; } \\
\text { Negociação de PDP por } \\
\text { laboratório e parceiros, e não PDP } \\
\text { por PDP; } \\
\text { Estabelecimento de manuais } \\
\text { específicos para medicamentos } \\
\text { biológicos; } \\
\text { Inclusão das demandas dos } \\
\text { estados; } \\
\text { de moção de políticas } \\
\text { acompanhamentomento e } \\
\text { contratações. }\end{array}$ \\
\hline
\end{tabular}

Fonte: elaboração própria a partir das respostas ao inquérito (2016).

O compromisso de compra dos produtos objetos de PDP pelo governo e a questão de investimentos foram os elementos facilitadores para execução das parcerias mais indicados pelos entrevistados $(n=4)$. Segundo um dos atores, o Programa para o Desenvolvimento do Complexo Industrial da Saúde (PROCIS) representa um elemento facilitador importante para as PDP de medicamento para DN e DCNT, desde que haja transparência na seleção de propostas e na destinação dos recursos. O PROCIS é coordenado pelo MS e visa estruturar e modernizar os produtores públicos por meio de investimentos na infraestrutura de produção e inovação destes. Entre os objetivos do Programa, está o fortalecimento das PDP para o desenvolvimento e a absorção de produtos estratégicos para 
o $\mathrm{SUS}^{20}$.

Os elementos dificultadores para execução dos projetos, segundo os entrevistados, variaram de acordo com a tecnologia. Para as PDP de medicamentos para DN, 60\% $(n=33)$ dos respondentes indicaram o valor de fornecimento como o elemento que mais desfavorece a execução dos projetos; $51 \%$, o custo produtivo; $45 \%$, os estudos clínicos; $38 \%$, quesitos regulatórios e plataforma produtiva; $21 \%$, a incorporação da tecnologia; $9 \%$, outros elementos; e 1\%, a propriedade intelectual. Já para a execução das PDP de medicamentos para DCNT, 59\% $(n=32)$ consideraram a plataforma produtiva e a incorporação da tecnologia como os fatores mais relevantes; $53 \%$, a propriedade intelectual; $40 \%$, o valor de fornecimento; $35 \%$, o custo produtivo, os estudos clínicos e os quesitos regulatórios; e 1\%, outros elementos.

Como facilitadores para execução dos projetos, a Portaria $n^{\circ}$ 2531/2014 foi considerada um dos elementos. Entretanto, como medidas auxiliares, foram indicados pontos de alteração nesse marco normativo, no que tange à definição da lista de produtos estratégicos para o SUS. Foi destacada por maior número de atores $(n=5)$ a vinculação de produtos de baixo e de alto valor agregado, fazendo combos ou cesta de produtos de PDP. Trata-se de apresentar, já nesta lista, aqueles medicamentos que deverão ser objeto de propostas conjuntas, ou seja, o proponente ao selecionar um produto para DCNT de alto valor agregado para se estabelecer uma PDP, obrigatoriamente estaria vinculado à apresentação de outra proposta de parceria para determinado medicamento para DN.

Outra medida apresentada neste sentido foi o monitoramento do horizonte tecnológico dos produtos a fim de se antever possíveis substituições de tecnologias, as quais não deveriam ser objeto de PDP.

As questões de preços diferenciados para produtos objeto de PDP e de investimentos nos produtores públicos também foram respostas recorrentes no inquérito como medidas auxiliares para a execução das PDP. Associadas a estas, figuraram-se as medidas de garantia de segurança às entidades produtoras envolvidas e do cumprimento dos compromissos do setor governamental.

\subsection{Relatório de caso individual da Parceria para o Desenvolvimento Produtivo de Rifampicina, Isoniazida, Etambutol e Pirazinamida para tuberculose}

A tuberculose é uma doença transmissível negligenciada de alta magnitude causada por uma micobactéria do tipo Mycobacterium tuberculosis que afeta prioritariamente os pulmões ${ }^{8,21}$. Segundo relatório da OMS, em 2014, foram 9,6 milhões novos casos em todo o mundo e 1,5 milhão de mortes em decorrência da tuberculose ${ }^{8}$. Apesar disto, a doença é tratável e curável.

Para o controle dessa doença e de outras comorbidades, foi instituído no Brasil o Programa Nacional de Controle da Tuberculose. No componente assistência desse Programa, o abastecimento ocorre, principalmente, por produtores públicos, os quais contribuíram com $84,6 \%$ da produção 
do elenco de medicamentos em $2013^{22}$.

Nesse elenco de medicamentos do Programa, foi incorporado em 2009 o medicamento dose fixa combinada Rifampicina $150 \mathrm{mg}$, Isoniazida $75 \mathrm{mg}$, Pirazinamida $400 \mathrm{mg}$ e Etambutol $275 \mathrm{mg}$ (4 em 1), na forma farmacêutica comprimido, conforme preconizado pela OMS e utilizado na maioria dos países para adultos e adolescentes ${ }^{23}$.

Nessa época, não havia fabricante desse produto no Brasil, bem como o produtor internacional não possuía registro sanitário na ANVISA. O fornecimento ao SUS ocorria mediante importação pelo Fundo Rotatório, uma vez que a ANVISA pode dispensar de registro medicamentos adquiridos por intermédio de organismos multilaterais internacionais para uso em programas de saúde pública pelo $\mathrm{MS}^{24}$.

Diante desse contexto, os quatro IFA usados para produção do medicamento foram incluídos na lista de produtos estratégicos para o SUS em 2008, conforme Portaria GM/MS n ${ }^{\circ}$ 978, a fim de sinalizá-los como candidatos potenciais para iniciativas voltadas ao incremento da produção local. Em 2009, como estratégia estruturante para regulação do abastecimento do produto, a PDP do 4 em 1 foi estabelecida por Farmanguinhos com a empresa Lupin Limited para a transferência de tecnologia e a capacitação para a produção pública nacional de novo medicamento no país. O TC entre MS e Farmanguinhos foi assinado na XI reunião ordinária do Grupo Executivo do Complexo Industrial da Saúde (GECIS), finalizando a fase I da PDP.

A entidade privada indiana Lupin Limited é a responsável pela transferência da tecnologia de fabricação do 4 em 1 para Farmanguinhos e pela produção dos IFA. No decorrer do projeto, o produtor público absorverá toda a tecnologia de produção do medicamento, iniciando pelas etapas menos complexas, como o envase e embalagem, e posteriormente, a formulação e a compressão (formação dos comprimidos). O controle de qualidade do medicamento e dos IFA também faz parte das primeiras etapas de transferência.

Segundo Rezende ${ }^{13}$, devido à complexidade da síntese dos IFA, especialmente da Rifampicina, a entidade farmoquímica nacional ainda não foi identificada nos documentos desta PDP, ou seja, não há parceiro privado para produção nacional dos quatro IFA. Porém, o entendimento do MS era de que o medicamento fosse produzido localmente o mais breve possível para fornecimento ao SUS para, assim, reduzir a dependência frente às importações. Entretanto, a produção local do medicamento ainda não foi iniciada.

A autora ${ }^{13}$ considera que existem três marcos que caracterizam a evolução dos projetos de PDP: (1) início da produção local do medicamento pelo parceiro privado a partir do registro sanitário do produto e fornecimento ao MS; (2) registro sanitário e produção pelo produtor público; e (3) pós-registro e produção do medicamento pelo produtor público com IFA nacional. A partir desses marcos, a autora calculou, em sua pesquisa, o total executado em relação ao planejado e o Índice 
Linear de Sucesso (ILS) das PDP estabelecidas em 2009 ${ }^{13}$. O ILS refere-se ao valor percentual executado frente ao idealizado considerando os anos transcorridos desde o anúncio da PDP até a data de análise. Para a PDP do 4 em 1, o total executado e o ILS eram de 0\% em 2013.

O registro sanitário do 4 em 1 foi obtido pelo produtor público em novembro de 2014 e é o único registro existente no Brasil para esse medicamento. O local de fabricação constante nesse registro é a empresa Lupin, localizada na Índia. No decorrer da PDP, na medida de evolução da transferência de tecnologia, Farmanguinhos peticionará alterações pós-registro. A primeira destas será para inclusão de local de embalagem no produtor público e, mais ao final do projeto, para inclusão de local de fabricação.

Os atrasos verificados na fase II da PDP foram decorrentes, principalmente, de entraves regulatórios enfrentados pelos parceiros, tendo em vista a formulação conjunta de quatro IFA, a qual não estava prevista, anteriormente, nas normativas sanitárias. Outra barreira foi o desenvolvimento do medicamento na forma farmacêutica comprimido dose fixa combinada.

Acredita-se que as discussões no âmbito do CTR de Farmanguinhos tenham sido também fundamentais para o êxito do processo de registro sanitário do $4 \mathrm{em} 1$. Tal Comitê é formado por representantes do produtor público, do MS e da ANVISA, e é destinado ao acompanhamento das atividades referentes ao desenvolvimento, à produção, ao registro e ao pós-registro do produto objeto de $\mathrm{PDP}^{25}$.

Uma vez habilitados a iniciar a fase III da PDP, a primeira compra do 4 em 1 no âmbito da parceria ocorreu em novembro de 2015 por Termo de Execução Descentralizada (TED) celebrado entre o MS e a Fiocruz com vigência de um ano e recursos da ordem de R $\$ 9$ milhões ${ }^{26}$. Como a data de publicação deste instrumento indica o início da contagem do prazo da fase III, foram gastos seis anos para a fase II.

Porém, as entregas do produto da Lupin a Farmanguinhos, bem como deste ao MS, não foram realizadas. Para efetivação do fornecimento do produto importado do parceiro indiano, é necessário que Farmanguinhos realize o controle de qualidade e a liberação dos lotes conforme preceitos sanitários. Assim, as etapas de transferência de metodologia analítica e de controle de qualidade precisam estar concluídas. Entretanto, conforme informado pelo MS pelo e-SIC, o produtor privado informou a Farmanguinhos, em novembro de 2015, a existência de mudanças de procedimentos na etapa analítica e em outros processos, o que ocasionou atrasos no início do processo de importação e, consequentemente, na reanálise do produto. Dessa forma, o MS precisou recorrer a aquisição via organismo internacional, Organização Pan-Americana da Saúde (OPAS), para fornecimento do medicamento em 2016 pelo fornecedor europeu Svizera, sem registro na ANVISA.

Assim, o abastecimento do SUS não foi efetivamente iniciado pelo produto da PDP, apesar da publicação do primeiro instrumento específico de compra e indicação do início da fase III da parceria. De qualquer modo, o projeto continua em execução e o produtor público terá até 
novembro de 2020 para atingir a fase IV, ou seja, internalizar a tecnologia.

Quanto à adequação da área produtiva para absorção da tecnologia de produção, Farmanguinhos obteve o CBPF em novembro de 2016 para a linha de sólidos não estéreis que compreende a forma farmacêutica comprimido, referente ao $4 \mathrm{em} \mathrm{1.} \mathrm{Dessa} \mathrm{forma,} \mathrm{o} \mathrm{produtor} \mathrm{público} \mathrm{está} \mathrm{apto} \mathrm{a} \mathrm{receber,}$ de seu parceiro privado, o conhecimento para fabricação do medicamento em suas instalações produtivas.

\subsection{Relatório de caso individual da Parceria para o Desenvolvimento Produtivo de Betainterferona 1A para esclerose múltipla}

A esclerose múltipla é uma DCNT incapacitante que requer tecnologia de alto custo e de alta transcendência. A Betainterferona 1A é um medicamento biológico de alto valor agregado indicado para o tratamento dessa doença e contemplado no Grupo 1A do CEAF nas concentrações de 6.000.000 UI (22 mcg) e 12.000.000 UI (44 mcg) solução injetável por seringa preenchida ${ }^{27}$.

A indicação da Betainterferona 1A como produto estratégico para o SUS se deu na lista publicada em 2010 pelo MS pela Portaria $n^{0}$ 1.284. Em tal lista, as interferonas constavam no grupo de produtos obtidos por rotas biológicas. A PDP desse produto foi celebrada em novembro de 2010 entre o MS e o Instituto de Tecnologia em Imunobiológicos (Biomanguinhos) da Fiocruz pelo TC $n^{0} 11 / 2010$, finalizando a fase I da parceria ${ }^{15}$.

Nessa época, havia um único registro sanitário de Betainterferona 1Anas concentrações utilizadas para o tratamento de esclerose múltipla. Esse registro, publicado em outubro de 2008, refere-se ao medicamento Rebif® da empresa Merck S/A, fabricado internacionalmente ${ }^{28}$. Portanto, a aquisição do produto da empresa pelo MS se dava por inexigibilidade de licitação.

A PDP de Betainterferona 1A trata-se de uma das primeiras parcerias de biológicos estabelecida. O seu estabelecimento visava, entre outros pontos, a diminuição da vulnerabilidade do SUS frente ao mercado internacional e a capacitação nacional na produção de medicamentos biológicos.

O parceiro privado indicado inicialmente para essa PDP foi o Aché Laboratórios. Entretanto, em 2012, com a criação da joint venture Bionovis S.A. - Companhia Brasileira de Biotecnologia Farmacêutica, da qual o Aché faz parte, juntamente à EMS, Hypermarcas e União Química, os parceiros foram alterados. Atualmente, a PDP conta com os parceiros privados Bionovis e Merck S.A ${ }^{15}$.

O acordo de transferência de tecnologia entre os parceiros foi assinado em setembro de $2015^{29}$. Foram, então, cinco anos para a implementação da proposta de PDP e do TC pelos parceiros. Essas atividades compreendem a fase II, de projeto de PDP. Como um dos parceiros privados era detentor do registro do produto, a conclusão dessas atividades permitia que a fase III pudesse ser iniciada. 
A primeira aquisição de Betainterferona 1A no âmbito da PDP ocorreu pelo TED no 94/2015 assinado pelo MS e Fiocruz em 27/11 e publicado no DOU de 30/11/201526. A partir dessa publicação, deu-se início à fase III da PDP.

Uma vez que a primeira compra da Betainterferona 1A se deu apenas com o registro do parceiro privado Merck, Biomanguinhos teve 60 dias para apresentar à ANVISA o pedido de registro em seu nome e, após um ano, só poderia fornecer o produto se obtivesse o registro sanitário. Tal registro foi obtido anteriormente ao prazo estabelecido na normativa, em 29 de fevereiro de 2016, com locais de fabricação internacionais - Ares Trading, Uruguai; Merck Serono, Suíça e Itália -, e nacional - Fiocruz, Rio de Janeiro. Assim, a segunda aquisição foi realizada em dezembro de 2016.

Os recursos financeiros envolvidos na primeira e na segunda compras do produto desta PDP foram da ordem de R $\$ 172$ milhões no período de dois anos ${ }^{26}$.A economia para os sete anos da fase III está prevista em R $\$ 27$ milhões segundo Biomanguinhos ${ }^{29}$.

O CBPF obtido por Biomanguinhos em março de 2015 corresponde à linha de embalagem secundária de produtos estéreis. Resta ainda a obtenção do certificado para a linha produtiva do IFA de Betainterferona 1A para absorção desta etapa produtiva. Segundo informações de Biomanguinhos ${ }^{29}$, o acordo de transferência de tecnologia tem duração de sete anos, ou seja, a internalização completa da tecnologia pelo Instituto deve ocorrer até 2022.

\subsection{Relatório de casos cruzados}

Os elementos que influenciam a execução das PDP são variáveis de acordo com o tipo de produto, da rota de síntese do fármaco, da indicação terapêutica e dos aspectos econômicos e sociais envolvidos na fabricação e no uso dos medicamentos. O Quadro 3 apresenta a análise comparativa das PDP do 4 em 1 para tuberculose e da Betainterferona 1A para esclerose múltipla segundo as variáveis estabelecidas.

Quadro 3- Análise comparativa das Parceria para o Desenvolvimento Produtivo (PDP) de Rifampicina, Isoniazida, Etambutol e Pirazinamida e Betainterferona 1A

\begin{tabular}{|c|c|c|c|c|}
\hline \multirow[t]{2}{*}{ Variáveis de análise } & & \multicolumn{2}{|c|}{ Casos } & Fonte \\
\hline & $\begin{array}{c}\text { Rifampicina, } \\
\text { Isoniazida, } \\
\text { Etambutol e } \\
\text { Pirazinamida } \\
\text { (4 em 1) }\end{array}$ & Betainterferona $\mathbf{1 A}$ & & \\
\hline \multicolumn{2}{|l|}{ Grupo de medicamento } & Doença negligenciada & $\begin{array}{l}\text { Doença crônica não } \\
\text { transmissível }\end{array}$ & MS \\
\hline \multicolumn{2}{|l|}{ Indicação terapêutica } & Tuberculose & Esclerose múltipla & MS \\
\hline \multicolumn{2}{|c|}{ Forma farmacêutica/ Apresentação } & $\begin{array}{l}\text { Comprimido/ } 150+75+ \\
275+400 \mathrm{mg}\end{array}$ & $\begin{array}{l}\text { Solução injetável/ seringa } \\
\text { preenchida } 22 \text { e } 44 \mathrm{mcg} \\
(6.000 .000 \text { e } 12.000 .000 \mathrm{UI})\end{array}$ & MS \\
\hline
\end{tabular}




\begin{tabular}{|c|c|c|c|c|}
\hline \multicolumn{2}{|l|}{ Variáveis de análise } & \multicolumn{2}{|c|}{ Casos } & Fonte \\
\hline \multicolumn{2}{|c|}{$\begin{array}{l}\text { Rota de síntese do insumo farmacêutico } \\
\text { ativo (IFA) }\end{array}$} & Química & Biológica & MS \\
\hline \multicolumn{2}{|l|}{ Produtor público } & Farmanguinhos & Biomanguinhos & MS \\
\hline \multicolumn{2}{|c|}{ Parceiro nacional desenvolvedor do IFA } & Não há & Bionovis & MS \\
\hline \multicolumn{2}{|c|}{ Parceiro desenvolvedor do medicamento } & Lupin - Índia & Merck Serono - Suíça, Itália & ANVISA \\
\hline \multicolumn{2}{|c|}{ Ano de aprovação da proposta } & 2009 & 2010 & MS \\
\hline \multicolumn{2}{|c|}{ Duração do projeto - fase II } & 6 anos & 5 anos & MS \\
\hline \multicolumn{2}{|c|}{ Fase do processo em 2016} & Fase III - PDP & Fase III - PDP & MS \\
\hline \multicolumn{2}{|c|}{$\begin{array}{l}\text { Data de início da fase III (publicação do } \\
\text { primeiro instrumento de compra) }\end{array}$} & $30 / 11 / 2015$ & $30 / 11 / 2015$ & DOU \\
\hline \multirow{2}{*}{$\begin{array}{l}\text { Data de obtenção do } \\
\text { registro sanitário }\end{array}$} & Privado & Não peticionado & $20 / 10 / 2008$ & ANVISA \\
\hline & Público & $13 / 11 / 2014$ & 29/02/2016 & ANVISA \\
\hline \multicolumn{2}{|c|}{$\begin{array}{l}\text { Certificado de Boas Práticas de Fabricação } \\
\text { (CBPF) - Linha/Validade }\end{array}$} & $\begin{array}{l}\text { Sólidos não estéreis } \\
\text { (Formulação e } \\
\text { embalagem) - 11/2018 }\end{array}$ & $\begin{array}{l}\text { Embalagem secundária de } \\
\text { produtos estéreis - 03/2017 }\end{array}$ & ANVISA \\
\hline \multicolumn{2}{|l|}{ Valor unitário - 2015} & $\mathrm{R} \$ 0,38$ & $\begin{array}{l}\mathrm{R} \$ 134,27(22 \mathrm{mcg}) \\
\mathrm{R} \$ 151,60(44 \mathrm{mcg})\end{array}$ & DOU \\
\hline \multicolumn{2}{|c|}{ Valor da primeira aquisição - 2015} & $\mathrm{R} \$ 9.157 .863,15$ & $\mathrm{R} \$ 84.192 .192,00$ & DOU \\
\hline \multicolumn{2}{|c|}{ Valor da segunda aquisição - 2016} & Não houve & $\mathrm{R} \$ 87.989 .408,00$ & DOU \\
\hline
\end{tabular}

Fonte: elaboração própria (2016).

Notas: DOU - Diário Oficial da União; MS - Ministério da Saúde.

Em relação à rota de síntese, os IFA para DN são, em grande parte, fármacos antigos, obtidos por rota produtiva de síntese química. O setor farmoquímico encontra-se estruturado no Brasil com empresas de pequeno porte, em sua maioria, e capacidade limitada para competitividade com os grandes produtores asiáticos ${ }^{30}$. Assim, os produtores públicos encontram dificuldades para a identificação de parceiros privados interessados na produção de IFA de síntese química para DN, o que está associado ao custo produtivo e ao valor de fornecimento.

Analisando as rotas de síntese e as parcerias estabelecidas, nota-se que, para a PDP do 4 em 1, Farmanguinhos não apresentou farmoquímicas nacionais para produção dos quatro IFA. Dessa forma, mesmo que o produtor público incorpore a tecnologia de produção, o país continuará dependente dos IFA fabricados internacionalmente.

O contrário ocorre na área de produtos biológicos. Apoiadas por projetos do Banco Nacional do Desenvolvimento Econômico e Social (BNDES) e Finep, com recursos próprios e de outras fontes, surgiram em meados de 2012 duas joint ventures nacionais no ramo biotecnológico: Bionovis SA e Orygen Biotecnologia SA. Esta última é formada atualmente por Biolab e Eurofarma. A Libbs Farmacêutica e o Laboratório Cristália, que antes também a compunham (no estabelecimento de algumas PDP), deixaram de integrá-la para montarem, em suas próprias unidades, plantas do ramo biotecnológico. Todas essas quatro empresas privadas nacionais têm parcerias estabelecidas com os produtores públicos para o desenvolvimento de produtos biológicos para DCNT. 
Associadas às empresas privadas nacionais estão entidades internacionais para condução do desenvolvimento dos produtos biológicos: Mabxience, empresa argentina, que se articula com a Libbs; Alteogen, coreana, em parceria com o Cristália; Pfizer, multinacional com sede nos Estados Unidos da América (EUA), em cooperação com Orygen; Janssen Cilag, belga; e Merck Serono, multinacional alemã, em parceria com a Bionovis. Além dessas empresas, há ainda, no ramo dos monoclonais, a empresa russa Biocad que estabeleceu PDP diretamente com o TECPAR, sem a presença de uma empresa privada brasileira. Algumas dessas empresas estão há mais tempo no mercado internacional e detêm expertise na área que é incipiente no Brasil.

Apesar de a PDP de Betainterferona 1A ter sido estabelecida junto às PDP de primeira geração, ou seja, junto ao lançamento da estratégia com parcerias de medicamentos sintéticos, somente veio a ser efetivamente executada quando se iniciaram as parcerias de segunda geração - de medicamentos biológicos e produtos para saúde - e se estabeleceram as novas empresas no ramo biotecnológico no país. Nesse período, Biomanguinhos solicitou alteração de seu parceiro privado nesta PDP.

A morosidade na fase II da PDP de Betainterferona 1A esteve associada, então, principalmente às bases contratuais da parceria e às alterações do detentor ou desenvolvedor da tecnologia. Após esses trâmites, conseguiu-se atingir a fase III, uma vez que um dos novos parceiros era o detentor do registro sanitário do produto.

No caso do 4 em 1, o grande tempo dispendido na fase de projeto deveu-se a questões regulatórias de registro de uma nova formulação dose fixa combinada no país, a qual não era registrada pelo parceiro privado na ANVISA e cujo regramento ainda não estava disposto pela Agência.

No que tange à adequação das plataformas produtivas, Farmanguinhos já possui linha produtiva completa com CBPF para produção do 4 em 1 na forma farmacêutica de comprimido, embora ainda não o possa produzi-lo por não ter absorvido etapas anteriores do processo, quais sejam: transferência de metodologia e controle de qualidade do medicamento e IFA. Para Biomanguinhos, que conta com novos parques produtivos, restam adequações para obtenção do CBPF da área de formulação de produto biológico e envase em apresentação injetável. Tal fato evidencia o elemento considerado, pelos entrevistados do inquérito, como o mais dificultador para execução de PDP para DCNT, a plataforma produtiva.

Como apontam Kalil et al. ${ }^{31}$, para realizar a absorção tecnológica de produtos biológicos de maior complexidade, os produtores públicos necessitam de reestruturação, o que demanda tempo e recursos. Os recursos vêm do próprio fornecimento do produto ao MS por meio da PDP e, quando necessário, de outras fontes adicionais de financiamento previstas no projeto.

Considerando a primeira aquisição dos produtos, o faturamento total dos parceiros para o atendimento anual das demandas do MS de Betainterferona 1A é mais de nove vezes superior ao valor total do 4 em 1. Comparando-se o valor unitário, o preço da menor concentração de 
Betainterferona 1A é mais que 350 vezes o valor de um comprimido do $4 \mathrm{em} 1$. Isto evidencia o elemento "valor de fornecimento" apresentado pelos entrevistados no inquérito como o mais dificultador para execução das PDP de medicamentos para DN.

Há ainda que se considerar que o primeiro fornecimento de 4 em 1 não se concretizou tecnicamente devido a falhas no planejamento e na comunicação entre parceiros e no gerenciamento da absorção da tecnologia. Analisando-se o contexto institucional, existem outras barreiras, como a natureza do produtor público, a cultura dos gestores não voltada para eficiência empresarial, a incapacidade para gerir projetos variados ao mesmo tempo e ainda a dificuldade em definir prioridades.

\section{DISCUSSÃO}

O estudo de casos múltiplos permitiu verificar os elementos que influenciam a execução das PDP, os quais são variáveis a depender da tecnologia envolvida e dos aspectos econômicos e sociais relacionados ao medicamento ou à patologia para a qual é utilizado, DN ou DCNT. Tal análise e as constatações apresentadas estão atreladas ao desempenho da estratégia de PDP, sendo necessário monitorá-la estrategicamente na Política Nacional de Saúde e identificar pontos de melhoria em sua execução e interação com as demais políticas e estratégias.

O quadro verificado de disputa por produtos biológicos para DCNT e o quase esquecimento de produtos de síntese química para DN pode ser decorrente da nova busca das indústrias farmacêuticas. Segundo Kalil et al. ${ }^{31}$, com o aumento da concorrência após a introdução dos genéricos de medicamentos de síntese química e a ameaça de sustentabilidade da indústria nacional a longo prazo, iniciou-se uma "nova corrida", em busca de produtos de alto valor agregado, como os biológicos.

Infere-se ainda que o baixo número de propostas recebidas de medicamentos para DN se deve ao baixo interesse da indústria farmacêutica nesses produtos, justificado pelo reduzido potencial de retorno lucrativo para a indústria, uma vez que a população atingida é de baixa renda e presente, em sua maioria, nos países em desenvolvimento 5 .

No que tange a novas propostas, há também um reduzido número de apresentação de projetos de produtos órfãos, assim denominados por não despertar o interesse produtivo de mais nenhuma empresa, nem mesmo de seu inventor. A exemplo, têm-se os medicamentos L-asparaginase e Dactinomicina, elencados na lista anual de produtos estratégicos para o SUS, para os quais não foram apresentadas propostas de PDP em 2015 ${ }^{17}$. A L-asparaginase é um medicamento indicado para leucemia linfoide aguda infantil que teve a comercialização interrompida em 2014 pelo único produtor com registro na ANVISA. O medicamento oncológico Dactinomicina, de igual forma, teve a comunicação de interrupção de produção em 2015 pelo seu único produtor. Esse cenário merece atenção e deve ser contornado para que possíveis episódios de desabastecimento futuro sejam evitados. 
Resgatando a teoria em que se baseou o estudo de casos múltiplos, o modelo de PDP que se tem atualmente no Brasil talvez não seja a estratégia mais adequada para a garantia do abastecimento de medicamentos para DN, tendo em vista o quadro verificado com as PDP nesse campo nos últimos anos: $4 \%$ do total de projetos estabelecidos, extinção de $75 \%$ das parcerias firmadas e entrega de medicamento por meio da única parceria vigente não concluída.

Assim, medidas podem ser necessárias para auxiliar a execução dos projetos e para atrair novas propostas no campo das DN, como: (a) a adequação da iniciativa a esse tipo de produto, a exemplo das sugestões, apresentadas pelos atores envolvidos com as PDP, de conjugação de produtos de alto e baixo valor agregado e de articulação a outros incentivos financeiros; e (b) a implantação de novas estratégias que, associadas às PDP, possam auxiliar no controle dos elementos dificultadores do processo de execução dos projetos.

Como apresenta $\mathrm{Gemal}^{14}$, faz-se necessário discutir formas para que o Estado brasileiro integre suas políticas públicas mantendo o equilíbrio entre os aspectos econômico e sanitário e definindo situações específicas para cada segmento: medicamentos de alto valor agregado e medicamentos para DN. As sugestões apresentadas pelos entrevistados para facilitar a execução das PDP podem auxiliar os gestores públicos na identificação desse ponto de equilíbrio.

A especialização dos produtores públicos em plataformas foi uma das medidas auxiliares para as PDP apresentadas no inquérito. Nesse sentido, a nova gestão do MS apresentou, em outubro de 2016 na reunião do GECIS, a denominada Política de Plataformas Inteligentes de Tecnologia em Saúde que congrega, além das PDP, as Compensações Tecnológicas (Off-Set) e Encomendas Tecnológicas $^{32}$. Tais ferramentas já eram utilizadas pelo MS e, agora, são anunciadas com o foco em plataformas tecnológicas: biotecnologia, doenças raras, fitoterápicos, DN, produtos para saúde, hemoderivados, medicina nuclear e síntese química. No campo das DN, o enfoque dado na apresentação do governo foi para os produtos tuberculostáticos e penicilínicos.

Embora não apontada pelos entrevistados, é importante destacar a estratégia prevista das PDP de pesquisa, desenvolvimento e inovação (PDI). Tal previsão está no marco normativo das PDP de 2014, porém sem data definida para conclusão. Em 2015, foi instituído um Grupo de Trabalho (GT) para elaboração de uma normativa para essa nova modalidade de parceria. Entre os principais pontos de discussão dessa pauta estava o estabelecimento de um rol de doenças ou condições de saúde para apresentação de propostas de PDP de PDI e a normatização do instrumento por meio de Portaria Interministerial do MS, MDIC e MCTIC ${ }^{33}$. Entretanto, conforme consulta realizada pelo e-Sic, este grupo de trabalho não se encontrava vigente em abril de 2017, tendo sido interrompidas suas atividades, sem finalização da elaboração da portaria.

Caso sejam retomadas as discussões e estabelecida a normativa nos moldes que estava sendo construída, essa estratégia pode ser relevante no campo de DN para o desenvolvimento de produtos inovadores, como é a tendência internacional de parcerias público-privadas ${ }^{34,35}$. 


\section{CONCLUSÃO}

A análise do estudo de casos múltiplos contribui para elucidar a teoria de que o modelo atual das PDP não está adequado para a garantia do abastecimento de medicamentos para DN. Alterações no formato dessa iniciativa, a fim de aprimorar o desempenho das parcerias nesse campo, são desejáveis para que o acesso a medicamentos estratégicos por parte da população seja concretizado. Algumas medidas para tal foram identificadas pelos atores envolvidos com as PDP e por esta pesquisa.

As limitações do estudo estão associadas à classificação dos processos de PDP como secretos pela Lei de Acesso à Informação e à não apresentação da teoria e dos achados de pesquisa para análise de outros especialistas. Entretanto, as informações públicas das parcerias disponibilizadas nos portais eletrônicos governamentais e as obtidas por meio do inquérito junto aos atores envolvidos com as PDP puderam demonstrar evidências para a teoria apresentada.

A análise dos casos, dos elementos facilitadores e dificultadores e das medidas auxiliares ao processo de estabelecimento das PDP, apresentados pelos entrevistados, permite o aprendizado institucional da iniciativa e pode contribuir para a tomada de decisões dos gestores públicos que coordenam a estratégia. Ademais, o estudo fornece conhecimento para novas pesquisas voltadas para a avaliação da implementação e do desempenho das PDP, em especial buscando soluções para DN.

\section{REFERÊNCIAS BIBLIOGRÁFICAS}

1. Paim JS. A Constituição Cidadã e os 25 anos do Sistema Único de Saúde (SUS). Cad Saúde Pública. 2013;29(10):1927-36.

2. Mendes EV. 25 anos do Sistema Único de Saúde: resultados e desafios. Estud Av. 2013;27(78):27-34.

3. Mendes EV. As redes de atenção à saúde. Ciênc Saúde Coletiva. 2010;15(5):2297-305.

4. Western Pacific Region, World Health Organization. Neglected tropical diseases [Internet] [data desconhecida]. [acesso em 18 out 2015]. Disponível em: http://www.wpro.who.int/topics/ neglected_tropical_diseases/en/

5. Ministério da Saúde (BR), Secretaria de Ciência, Tecnologia e Insumos Estratégicos, Departamento de Ciência e Tecnologia. Doenças negligenciadas: estratégias do Ministério da Saúde. Rev Saúde Pública. 2010;44(1):200-2.

6. Garcia LP, Magalhães LCG de, Áurea AP, Santos CF dos, Almeida RF de. Epidemiologia das doenças negligenciadas no Brasil e gastos federais com medicamentos [Internet]. IPEA. 2011. [acesso em 18 out 2015]. Disponível em: http://www.ipea.gov.br/portal/index.php?option=com ISSN 1982-8829 Tempus, actas de saúde colet, Brasília, 11(4), 147-169, dez, 2017. Epub Ago/2018 
content\&view $=$ article $\&$ id $=8064$

7. Ministério da Saúde (BR), Secretaria de Ciência, Tecnologia e Insumos Estratégicos, Departamento de Ciência e Tecnologia. Caderno 2: doenças negligenciadas [Internet]. [acesso em 18 out 2015]. Disponível em: http://bvsms.saude.gov.br/bvs/publicacoes/Pesquisa_Saude/ tela3_2.html

8. World Health Organization. World Health Statistics 2016: monitoring health for the SDGs, sustainable development goals [Internet]. 2016 [acesso em 21 nov 2016]. Disponível em: http://www.who.int/mediacentre/news/releases/2016/health-inequalities-persist/en/

9. Ministério da Saúde (BR), Secretaria de Vigilância em Saúde, Departamento de Análise de Situação de Saúde. Plano de ações estratégicas para o enfrentamento das doenças crônicas não transmissíveis (DCNT) no Brasil 2011-2022 [Internet]. Brasília: MS; 2011. 160 p. (B. Textos Básicos de Saúde). [acesso em 10 abr 2016]. Disponível em: http://bvsms.saude.gov.br/bvs/ publicacoes/plano_acoes_enfrent_dent_2011.pdf

10. Organização das Nações Unidas-Brasil. Objetivo do Desenvolvimento do Milênio (ODS3) [Internet]. 2015 [acesso em 10 dez 2016]. Disponível em: https://nacoesunidas.org/ pos2015/ods3/

11. Sundfeld CA, Souza RP de. Parcerias para o desenvolvimento produtivo em medicamentos e a Lei de Licitações. Rev Direito Adm. 2013;264(0):91-133.

12. Fonseca EM da, Costa NR. Federalismo, complexo econômico-industrial da saúde e assistência farmacêutica de alto custo no Brasil. Ciênc Saúde Coletiva. 2015;20(4):1165-76.

13. Rezende KS. As parcerias para o desenvolvimento produtivo e estímulo à inovação em instituições farmacêuticas públicas e privadas [Dissertação] [Internet]. Rio de Janeiro: Escola Nacional de Saúde Pública Sérgio Arouca (ENSP); 2013 [citado 18 de outubro de 2015]. Disponível em: http://157.86.8.70:8080/certifica/bitstream/icict/2367/2/0000041.pdf

14. Gemal AL. Estudo de Caso: Produção de medicamentos para o tratamento da tuberculose no Brasil. Recomendações visando ao aprimoramento da capacidade nacional de produção de medicamentos para a tuberculose. [Internet]. Rio de Janeiro: Ministério da Saúde em parceria com a Fundação Ataulpho de Paiva; 2011 [acesso em 29 de dezembro de 2015] p. 51. Disponível em: http://www.fundacaoataulphodepaiva.com.br/_arq/BILL\%20GATES/2014/Andre_Gemal_ versao_final.pdf

15. Ministério da Saúde (BR). Propostas de projeto de PDP aprovadas de 2009 a 2014 [Internet]. [acesso em 3 mar 2016]. Disponível em: http://portalsaude.saude.gov.br/images/pdf/2016/ fevereiro/16/Consolidado-PDP-2009-a-2014---no-site-em-16.02.2016.pdf 
16. Brasil. Ministério da Saúde. Portaria n ${ }^{\circ} 2.531$, de 12 novembro de 2014. Redefine as diretrizes e os critérios para a definição da lista de produtos estratégicos para o Sistema Único de Saúde (SUS) e o estabelecimento das Parcerias para o Desenvolvimento Produtivo (PDP) e disciplina os respectivos processos de submissão, instrução, decisão, transferência e absorção de tecnologia, aquisição de produtos estratégicos para o SUS no âmbito das PDP e o respectivo monitoramento e avaliação [Internet]. Brasília, 13 nov 2014 [acesso em 29 jan 2016]. Disponível em: http://bvsms.saude.gov.br/bvs/saudelegis/gm/2014/prt2531_12_11_2014.html

17. Ministério da Saúde (BR). Balanço das Parcerias para o Desenvolvimento Produtivo (PDP) [Internet]. 9a Reunião Ordinária do Grupo Executivo do Complexo Industrial da Saúde GECIS; 2015 [acesso em 3 mar 2016]; Brasília. Disponível em: http://portalsaude.saude.gov.br/ images/pdf/2015/julho/06/Apresentacao-Balanco-PDP.pdf

18. Severino AJ. Metodologia do trabalho científico. São Paulo: Cortez; 2007.

19. Yin RK. Estudo de caso: planejamento e métodos. 2a ed. Porto Alegre: Bookman; 2001.

20. Brasil. Ministério da Saúde. Portaria n ${ }^{\circ}$ 506, de 21 de março de 2012. Institui o Programa para o Desenvolvimento do Complexo Industrial da Saúde (PROCIS) e seu Comitê Gestor [Internet]. Brasília, 22 mar 2012 [acesso em 20 dez 2015]. Disponível em: http://bvsms.saude.gov.br/ bvs/saudelegis/gm/2012/prt0506_21_03_2012.html

21. Ministério da Saúde (BR). Tuberculose [Internet]. [acesso em 21 de nov 2016]. Disponível em: http://portalms.saude.gov.br/saude-de-a-z/tuberculose

22. Santiago EMD. Produção Pública para os Componentes da Assistência Farmacêutica-2003 a 2013 [Dissertação] [Internet]. Brasília: Universidade de Brasília; 2015 [acesso em 2 out 2016]. Disponível em: http://repositorio.unb.br/handle/10482/18889

23. Ministério da Saúde (BR), Secretaria de Vigilância em Saúde, Departamento de Vigilância Epidemiológica. Tratamento diretamente observado (TDO) da tuberculose na atenção básica: protocolo de enfermagem. Brasília: Ministério da Saúde; 2011. 168 p. (Série F. Comunicação e Educação em Saúde).

24. Brasil. Lei no 9.782, de 26 de janeiro de 1999. Define o Sistema Nacional de Vigilância Sanitária, cria a Agência Nacional de Vigilância Sanitária, e dá outras providências [Internet]. Brasília, 27 jan 1999. [acesso em 28 mar 2017]. Disponível em: http://www.planalto.gov.br/ccivil_03/leis/L9782.htm

25. Ministério da Saúde (BR). Parceria para o Desenvolvimento Produtivo - PDP [Internet]. [acesso em 18 mar 2016]. Disponível em: http://portalsaude.saude.gov.br/index.php/o-ministerio/ principal/leia-mais-o-ministerio/581-sctie-raiz/deciis/12-deciis/12090-parceria-para-o-desenvolvi- 
mento-produtivo-pdp

26. Ministério da Saúde (BR), Secretaria de Ciência, Tecnologia e Insumos Estratégicos. Aquisições de produtos de Parcerias para o Desenvolvimento Produtivo (PDP) [Internet]. 2016. [acesso em 10 out 2016]. Disponível em: http://portalsaude.saude.gov.br/images/pdf/2016/setembro/05/Aquisi----es-de-produtos-de-PDP-no-site-em-01.09.2016.pdf

27. Brasil. Ministério da Saúde. Portaria $n^{0} 1.554$, de 30 julho de 2013. Dispõe sobre as regras de financiamento e execução do Componente Especializado da Assistência Farmacêutica no âmbito do Sistema Único de Saúde (SUS) [Internet]. Brasília, 30 jul 2013 [acesso em 29 jan 2016]. Disponível em: http://portalarquivos2.saude.gov.br/images/pdf/2014/dezembro/30/ Anexos-i-ii-iii-iv-v-vi-pt-gm-ms-1554-2013-JANEIRO-2015-atualizada.pdf

28. Anvisa (BR). Consultas - Detalhes do Produto: REBIF [Internet]. 2008. [acesso em 14 jan 2017]. Disponível em: http://consultas.anvisa.gov.br/\#/medicamentos/25351372466200811/

29. Schueler P. Bio produzirá o Betainterferona 1a [Internet]. Instituto de Tecnologia em Imunobiológicos. Bio-Manguinhos. Fiocruz. 2015 [acesso em 20 nov 2016]. Disponível em: https:// www.bio.fiocruz.br/index.php/noticias/1057-assinado-acordo-para-transferencia-de-tecnologiado-betainterferona-1a

30. Clipping. Há espaços competitivos para a indústria farmoquímica brasileira? [Internet]. Abiquifi; 2015. [acesso em 9 mar 2017]. Disponível em: Http://Abiquifi.Org.Br/Artigos/Ha-Espacos-competitivos-para-a-industria-farmoquimica-brasileira/

31. Kalil J, Moreira MAE, Tolentino RP. Transferência de tecnologia no Brasil: duas décadas de incorporações tecnológicas e lições aprendidas com os produtos biológicos. Divulg Em Saúde Para Debate. 2013;(50):84-92.

32. Ministério da Saúde (BR), Secretaria de Ciência, Tecnologia e Insumos Estratégicos. Política de Plataformas Inteligentes de Tecnologias para Saúde [Internet]. 12a Reunião do Grupo Executivo do Complexo Industrial da Saúde; 2016. Brasília, DF. [acesso em 19 mar 2017]. Disponível em: http://portalsaude.saude.gov.br/index.php/o-ministerio/principal/leia-mais-o-ministerio/581-sctie-raiz/deciis/12-deciis/12076-grupo-executivo-do-complexo-industrial-da-saude-gecis

33. Ministério da Saúde (BR). Grupo de trabalho (GT) "Marco Regulatório das Parcerias de Pesquisa, Desenvolvimento e Inovação (PDI)" [Internet]. 9a Reunião Ordinária do Grupo Executivo do Complexo Industrial da Saúde - GECIS; 2015. Brasília/DF. [acesso em 23 jan 2017]. Disponível em: http://portalsaude.saude.gov.br/images/pdf/2015/julho/06/GT-PDI.pdf

34. Jannuzzi AHL. Sistemas de direitos de propriedade intelectual Sui Generis na indústria farmacêutica: um mecanismo de incentivo à inovação para doenças negligenciadas no Brasil? 
$169 / /$

[Tese] [Internet]. Rio de Janeiro: Universidade Federal do Rio de Janeiro; 2015. [acesso em 2 out 2016]. Disponível em: http://repositorio.int.gov.br:8080/jspui/handle/123456789/522

35. Jakobsen PH, Wang MW, Nwaka S. Innovative partnerships for drug discovery against neglected diseases. PLoSNegl Trop Dis. 2011;5(9):e1221.

Artigo apresentado em 24/04/17

Artigo aprovado em 11/02/18

Artigo publicado no sistema em 09/08/18 